(C) Revista de Matemática: Teoría y ApliCACiones 2020 27(1) : 73-92

CIMPA - UCR ISSN: 1409-2433 (PRINT), 2215-3373 (ONLINE)

DOI: https://doi.org/10.15517/rmta.v27i1.39949

\title{
ASSESSING THE INVASION SPEED OF TRIATOMINE POPULATIONS, CHAGAS DISEASE VECTORS
}

\section{EVALUACIÓN DE LA VELOCIDAD DE INVASIÓN DE POBLACIONES DE VECTORES TRIATOMINOS DE LA ENFERMEDAD DE CHAGAS}

\author{
Tewfik MahdjouB* Christopher M. KRIBS ${ }^{\dagger}$
}

Received: 12/May/2019; Revised: 20/Jun/2019;

Accepted: 5/Aug/2019

Revista de Matemática: Teoría y Aplicaciones is licensed under a Creative Commons Reconocimiento-NoComercial-Compartirigual 4.0 International License. Creado a partir de la obra en http://www.revistas.ucr.ac.cr/index.php/matematica

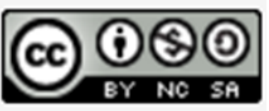

${ }^{*}$ University Abou Bekr Belkaïd, Research Laboratory: Nonlinear Analysis and Applied Mathematics, Tlemcen, Algeria. E-Mail: tewfik.mahdjoub@univ-tlemcen.dz

${ }^{\dagger}$ University of Texas at Arlington, Department of Mathematics, Arlington TX 76012, USA. E-Mail: kribs@uta.edu 


\begin{abstract}
Spraying insecticides to control triatomine populations, the vectors of Chagas disease, does not prevent the disease's reemergence in infested areas. Mathematical models try to explain this reemergence in terms of the factors underlying sylvatic transmission of the parasite Trypanosoma cruzi. The presence of reservoir hosts such as woodrats is essential to the infection's geographical spread. This study models a vector-host system using integrodifference equations to incorporate dispersal as well as hostvector interactions. These equations capture, simultaneously, the three processes taking place between successive generations: demography, infection and spatial dispersal. Travelling waves, the solutions of the integrodifference equations thus derived, allow one to calculate numerically the invasion speed of the disease. Neubert-Caswell's theorem can then be applied to calculate the analytical invasion speed.
\end{abstract}

Keywords: Chagas disease; vector-host contacts; integrodifference equations; travelling waves; invasion speed.

\title{
Resumen
}

La aplicación de insecticidas para controlar las poblaciones de triatominas, los vectores de la enfermedad de Chagas, no impide el resurgimiento de la enfermedad en zonas infestadas. Los modelos matemáticos tratan de explicar este resurgimiento en términos de los factores subyacentes a la transmisión sylvática del parásito Trypanosoma cruzi. La presencia de hospederos de reservorio como los ratas cambalacheras es esencial para la propagación geográfica de la infección. Este estudio modela un sistema vector-hospedero utilizando ecuaciones de integrodiferencia para incorporar las interacciones de dispersión y entre vector y hospedero. Estas ecuaciones capturan, simultáneamente, los tres procesos que se producen entre generaciones sucesivas: demografía, infección y dispersión espacial. Las ondas viajeras, las soluciones de las ecuaciones de integrodiferencia asíderivadas, permiten calcular numéricamente la velocidad de invasión de la enfermedad. El teorema Neubert-Caswell se puede aplicar para calcular la velocidad de invasión analítica.

Palabras clave: enfermedad de Chagas; interacción vector-hospedero; ecuaciones de integrodiferencias; ondas viajeras; velocidad de invasión.

Mathematics Subject Classification: 92D30. 


\section{Introduction}

Chagas disease, or American trypanosomiasis, is a disease that currently affects an estimated human population of 10 million people [7]. In 2008, approximately 14,000 deaths were attributed to this disease, which is no longer confined to Latin America. Its spread, mainly due to the high frequency of human mobility, organ donation, blood transfusions and vertical transmission (from infected mother to child), is such that the United States of America, Canada, parts of Europe (particularly Spain) and many Asian countries including Japan, are among the affected areas [4].

Although the causative agent of the disease, Trypanosoma cruzi, was discovered one hundred years ago, there is no vaccine to stop the spread of the disease, and the only solution advocated by the WHO is the fight against its vectors, which are blood-sucking bugs of the genus Triatoma. If these bugs live in the nests of mammals or birds, then they are said to be sylvatic. If they live in shelters neighboring human habitations, then they are domestic. In endemic areas, international control programs are based on the mass spraying of insecticides in domestic and peridomestic settings, whereas prevention programs more broadly are based on blood transfusion security by systematic epidemiological testing [3]. These program objectives have been only partially achieved because the disease re-emerges locally, supported by sylvatic transmission of the parasite. Not captured by means of laboratory studies, these transmission processes are an important focus for mathematical modeling studies.

The life cycle of $T$. cruzi includes intermediate hosts, or vectors, and definitive hosts, which are usually mammals. In the south of the United States, two vectors are dominant: Triatoma sanguisuga and Triatoma gerstaeckeri, associated, respectively, with raccoons (and opossums) and with woodrats [2]. Spatial dispersion plays an important role in triatomines' life cycle as it is essentially linked to foraging. The frequency of blood meals, which is very variable, has a major impact on the demography of the population. Indeed, it is established that spawning takes place after the blood meal. It is therefore appropriate to assume that between two successive generations three separate processes take place: demographics, infection and spatial dispersal.

This study develops a mathematical model which incorporates vector and host dispersal (as well as demography and infection) in order to describe the speed with which sylvatic $T$. cruzi infection spreads geographically. The mathematical model developed here uses integrodifference equations. To date the only other model developed to assess the invasion speed of sylvatic T. cruzi infection used a metapopulation model based on a large grid [1].

Rev.Mate.Teor.Aplic. (ISSN print: 1409-2433; online: 2215-3373) Vol. 27(1): 73-92, Jan-Jun 2020 


\section{Mathematical model}

Triatomines' life cycle is composed of seven stages: an egg stage, five instars, and an adult stage. Adults' longevity is usually longer than the accumulated times of the egg stage and larval stages [5]. We consider then vectors in two stages: juveniles and adults, while no age structure is imposed on the hosts. Two stages of infection are considered: susceptible and infected. The biological system thus consists of six classes (three susceptible and three infected). It is assumed to go through three processes during each time step: the demographic process between $[t, t+1 / 3]$; the infection process between $[t+1 / 3, t+2 / 3]$ and the dispersal processes between $[t+2 / 3, t+1]$. Dividing the interval $[t, t+1]$ into three equal intervals is formal: one process can be longer than another. The order of the process is the natural order: after the blood meal, the triatomines initiate their demography and transmit or receive T. cruzi. After that, they will disperse until the next blood meal.

\subsection{Population status before the infection}

Before the onset of the disease, the population is characterized by the state vector $N_{s}(x, t)=\left(V_{j s}, V_{a s}, H_{s}\right)^{T}(x, t)$ which represents respectively the juvenile vectors, adult vectors and hosts with, respectively, survival proportions (intuitively similar to probabilities) of $\sigma_{j s}, \sigma_{a s}$ and $\sigma_{h s}$. The transition proportion from juvenile to adult is $\tau_{s}$. Adult vector and host fertilities are denoted by $f_{v s}$ and $f_{h s}$, respectively (Figure 1).

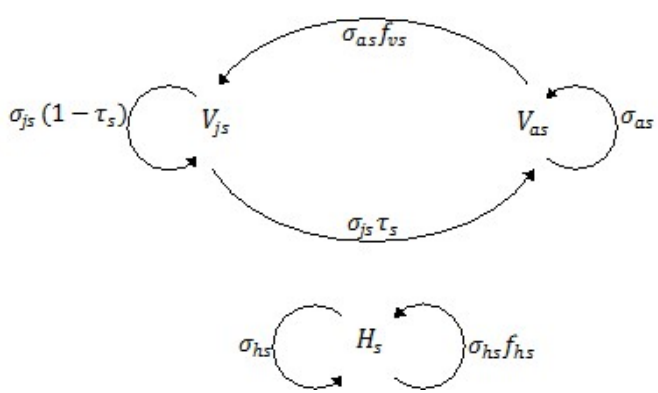

Figure 1: Vectors' and hosts' life cycle, between two successive generations, during the time interval $[t, t+1 / 3]$. 
In the simplest case, spatial dispersal can be described by linear movement along the real line $\mathbb{R}$. Prior to infection, the populations are assumed to have developed uniformly along the line, so that dispersal is balanced as well, making $N_{s}(x, t+1)=N_{s}(x, t+2 / 3)=N_{s}(x, t+1 / 3)$ (without change from infection or dispersal), and the biological system can be written in terms of just the demographic equations. Thus, for any habitat point $x$ and for any generation $t$,

$$
\begin{aligned}
V_{j s}(x, t+1) & =V_{j s}(x, t) \sigma_{j s}\left(1-\tau_{s}\right)+V_{a s}(x, t) \sigma_{a s} f_{v s} \\
V_{a s}(x, t+1) & =V_{j s}(x, t) \sigma_{j s} \tau_{s}+V_{a s}(x, t) \sigma_{a s} \\
H_{s}(x, t+1) & =H_{s}(x, t) \sigma_{h s}\left(1+f_{h s}\right)
\end{aligned}
$$

Healthy populations (vectors and hosts) are assumed to be in steady state. This steady state requires that vectors' and hosts' fertility are density dependent, so that we take:

$$
\begin{aligned}
f_{v s}=f_{v s}\left(V_{j s}, V_{a s}\right) & =f_{v}^{\max } \exp \left[-\left(V_{j s}+V_{a s}\right)\right], \\
f_{h} & =f_{h}^{\text {max }} \exp \left(-H_{s}\right),
\end{aligned}
$$

where $f_{v}^{\max }$ and $f_{h}^{\max }$ are the respective maximum fecundities. Steady state is achieved if demographic parameters satisfy the equations:

$$
\begin{aligned}
V_{j}^{*} & =\frac{1-\sigma_{a s}}{1-\sigma_{a s}+\sigma_{j s} \tau_{s}} \ln \frac{\sigma_{j s} \sigma_{a s} \tau_{s} f_{v}^{\max }}{\left(1-\sigma_{j s}+\sigma_{j s} \tau_{s}\right)\left(1-\sigma_{a s}\right)} \\
V_{a}^{*} & =\frac{\sigma_{j s} \tau_{s}}{1-\sigma_{a s}+\sigma_{j s} \tau_{s}} \ln \frac{\sigma_{j s} \sigma_{a s} \tau_{s} f_{v}^{\max }}{\left(1-\sigma_{j s}+\sigma_{j s} \tau_{s}\right)\left(1-\sigma_{a s}\right)}, \\
H_{s}^{*} & =\ln \frac{\sigma_{h s} f_{h}^{\max }}{1-\sigma_{h s}}
\end{aligned}
$$

After the infection's onset, three new classes appear: they are infected juvenile vectors, infected vector adults and infected hosts, whose densities are denoted, respectively, by $V_{j i}(x, t), V_{a i}(x, t)$ and $H_{i}(x, t)$. The total population is described then by the state vector $N(x, t)=\left(V_{j s}, V_{a s}, H_{s}, V_{j i}, V_{a i}, H_{i}\right)^{T}(x, t)$. The three processes that occur during each time step are described in the remainder of the section. 


\subsection{Demographic process}

Fecundity, which is a major life history trait, is initiated after the blood meal. We will assume in this study that infection affects demographic parameters of neither vectors nor hosts. If $\sigma_{j i}, \sigma_{a i}$ and $\sigma_{h i}$ represent respectively the survival proportions (at each time step) of infected juvenile vectors, adult vectors and hosts, then we have the equalities: $\sigma_{j i}=\sigma_{j s}=\sigma_{j}, \sigma_{a i}=\sigma_{a s}=\sigma_{a}$, and $\sigma_{h i}=\sigma_{h s}=\sigma_{h}$. The same hypothesis is applied to the transition proportion of infected juvenile vectors to reach adulthood, so $\tau_{i}=\tau_{s}=\tau$. Also, fecundity of infected adult vectors and hosts is not affected by infection: $f_{v i}=f_{v s}=f_{v}$ and $f_{h i}=f_{h s}=f_{h}$.

To highlight the parental effect, we distinguish between the new offspring (to vectors or hosts) by putting them in new temporary classes: new births to the vectors are placed in the class $V_{j}^{1}\left(\operatorname{resp} V_{j}^{2}\right)$ if they come from susceptible parents (resp. infected parents). Similarly $H_{1}$ (resp $H_{2}$ ) are the new classes of the new births to hosts if they come from susceptible parents (resp. infected). If we assume that the rate of vertical transmission of the disease in the vectors is null, then the classes $V_{j}^{1}$ and $V_{j}^{2}$ will furnish the juvenile susceptible class $V_{j s}$.

Assuming that vertical transmission among hosts affects a proportion $v$ of newborns, then $v H_{2}$ individuals go to the class $H_{i}$ and the other, non-infected, go to the class of susceptible hosts. Newborns to susceptible hosts are susceptible.

\subsection{Infection process}

Formally, the infection process takes place during the time interval $[t+1 / 3, t+2 / 3]$. Let $\Lambda_{j}, \Lambda_{a}$ and $\Lambda_{h}$ denote the proportions of infection of juvenile vectors, adult vectors and hosts, respectively. It is natural to assume that these proportions depend on the whole population state vector $N(x, t)$ and transmission rates of the disease between different classes which are entries of the WAIFW matrix (who acquires infection from whom).

If $\beta_{h j}, \beta_{h a}, \beta_{j h}$ and $\beta_{a h}$ represent the rate of transmission of infection from hosts to juvenile vectors, from hosts to adult vectors, from juvenile vectors to hosts and from adult vectors to hosts, respectively, then these proportions of infection are:

$$
\begin{aligned}
& \Lambda_{j}(N)=1-\exp \left(-\beta_{h j} H_{i}\right) \\
& \Lambda_{a}(N)=1-\exp \left(-\beta_{h a} H_{i}\right) \\
& \Lambda_{h}(N)=1-\exp \left(-\beta_{j h} V_{j i}-\beta_{a h} V_{a i}\right) .
\end{aligned}
$$


For simplicity, we consider that $\beta_{h j}=\beta_{j h}=\beta_{1}$ and $\beta_{h a}=\beta_{a h}=\beta_{2}$. At the end of the infection process, the balance, at each habitat point $x$, can be obtained via the transition matrix:

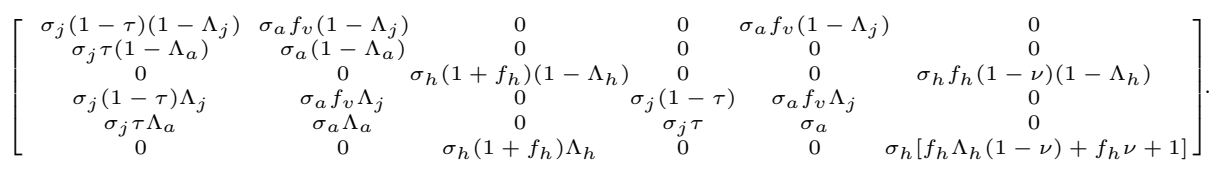

The demographic and infection processes are summarized in Figure 2.

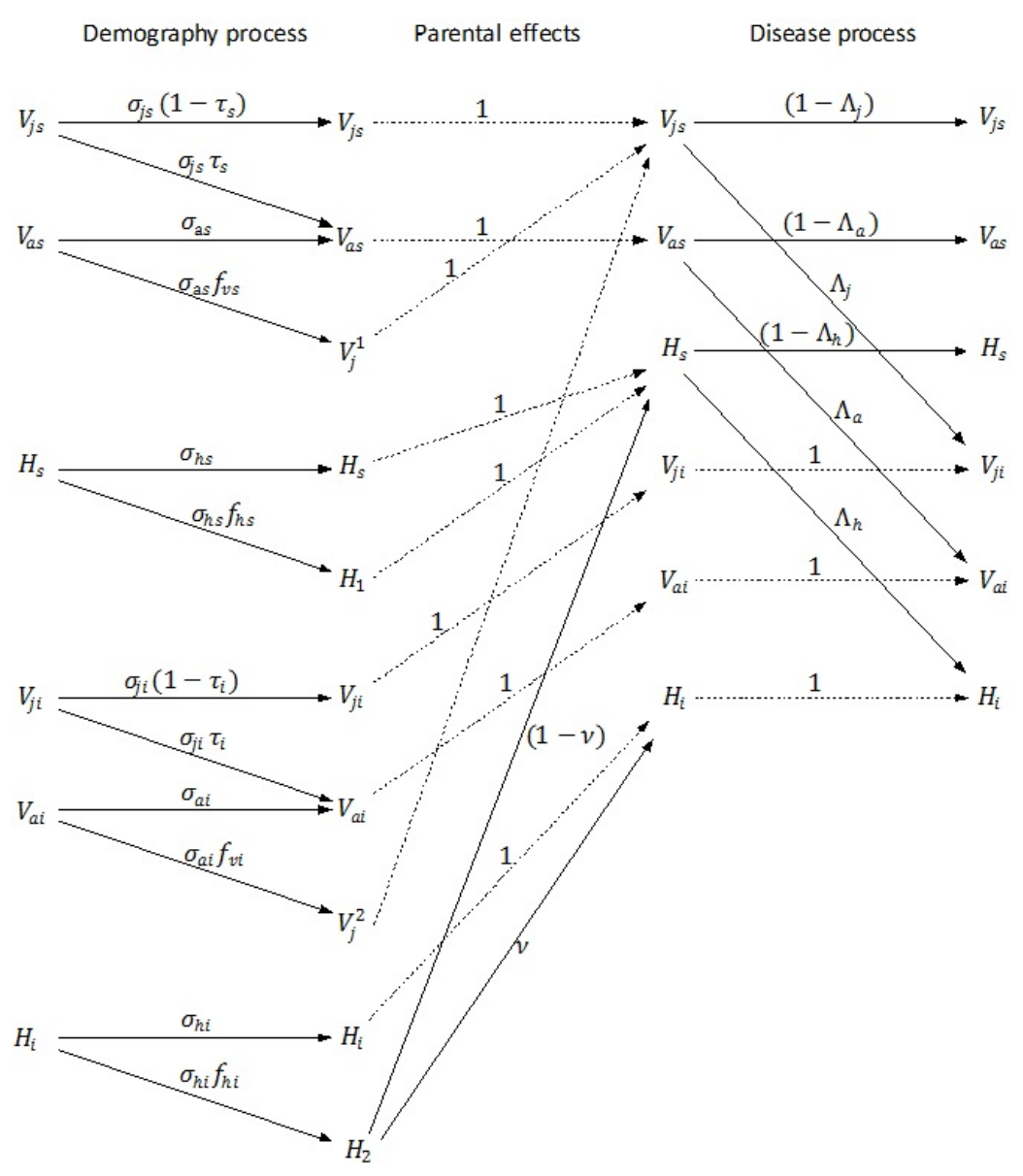

Figure 2: Schema of the demographic and infection processes, highlighting (between them) parental effects such as vertical transmission. 


\subsection{Dispersal process}

The dispersal process, corresponding to the search of blood meals for adult vectors, concerns also juvenile vectors. Laboratory results show that triatomine larvae are also able to disperse. On the other hand, dispersion among hosts is also permitted.

We assume that all species displace in one-dimensional space and the proportion of far dispersal distance decreases with distance. The Laplace kernel, defined by

$$
K(x)=\frac{1}{2 \alpha} \exp \left(-\frac{|x|}{\alpha}\right)
$$

where $\alpha$ is the mean dispersal ability, expressed as a (distance)/(time step), fits correctly with this biological property. Let $j, a$ and $h$, respectively, denote the mean dispersal abilities of juveniles, adults and hosts. If we denote by $K_{j}(x)$, $K_{a}(x)$ and $K_{h}(x)$ the dispersal kernels of juveniles, adults and hosts respectively and assume the disease does not affect these abilities, the densities are written as:

$$
\begin{aligned}
V_{j s}(x, t+1)= & \sigma_{j}(1-\tau) \int_{-\infty}^{+\infty}\left(1-\Lambda_{j}\right) K_{j}(|x-y|) V_{j s}(y, t) d y \\
& +\sigma_{a} \int_{-\infty}^{+\infty} f_{v}\left(1-\Lambda_{j}\right) K_{a}(|x-y|) V_{a s}(y, t) d y \\
& +\sigma_{a} \int_{-\infty}^{+\infty} f_{v}\left(1-\Lambda_{j}\right) K_{a}(|x-y|) V_{a i}(y, t) d y \\
V_{a s}(x, t+1)= & \sigma_{j} \tau \int_{-\infty}^{+\infty}\left(1-\Lambda_{a}\right) K_{j}(|x-y|) V_{j s}(y, t) d y \\
& +\sigma_{a} \int_{-\infty}^{+\infty}\left(1-\Lambda_{a}\right) K_{a}(|x-y|) V_{a s}(y, t) d y \\
H_{s}(x, t+1)= & \sigma_{h} \int_{-\infty}^{+\infty}\left(1+f_{h}\right)\left(1-\Lambda_{h}\right) K_{h}(|x-y|) H_{s}(y, t) d y \\
& +\sigma_{h}(1-\nu) \int_{-\infty}^{+\infty} f_{h}\left(1-\Lambda_{h}\right) K_{h}(|x-y|) H_{i}(y, t) d y
\end{aligned}
$$




$$
\begin{aligned}
V_{j i}(x, t+1)= & \sigma_{j}(1-\tau) \int_{-\infty}^{+\infty} \Lambda_{j} K_{j}(|x-y|) V_{j s}(y, t) d y \\
& +\sigma_{a} \int_{-\infty}^{+\infty} f_{v} \Lambda_{j} K_{a}(|x-y|) V_{a s}(y, t) d y \\
& +\sigma_{j}(1-\tau) \int_{-\infty}^{+\infty} K_{j}(|x-y|) V_{j i}(y, t) d y \\
& +\sigma_{a} \int_{-\infty}^{+\infty} f_{v} \Lambda_{j} K_{a}(|x-y|) V_{a i}(y, t) d y \\
V_{a i}(x, t+1)= & \sigma_{j} \tau \int_{-\infty}^{+\infty} \Lambda_{a} K_{j}(|x-y|) V_{j s}(y, t) d y \\
& +\sigma_{a} \int_{-\infty}^{+\infty} \Lambda_{a} K_{a}(|x-y|) V_{a s}(y, t) d y \\
& +\sigma_{j} \tau \int_{-\infty}^{+\infty} K_{j}(|x-y|) V_{j i}(y, t) d y \\
& +\sigma_{a} \int_{-\infty}^{+\infty} K_{a}(|x-y|) V_{a i}(y, t) d y \\
= & \sigma_{h} \int_{-\infty}^{+\infty}\left(1+f_{h}\right) \Lambda_{h} K_{h}(|x-y|) H_{s}(y, t) d y \\
& +\sigma_{h} \int_{-\infty}^{+\infty}\left[f_{h} \Lambda_{h}(1-v)+f_{h} v+1\right] K_{h}(|x-y|) H_{i}(y, t) d y
\end{aligned}
$$

But each point of the habitat is supposed to be at its equilibrium state, defined by equations (1), so that

$$
\begin{aligned}
\forall x \forall t \quad V_{j s}(x, t)+V_{j i}(x, t) & =V_{j}^{*}, \\
V_{a s}(x, t)+V_{a i}(x, t) & =V_{a}^{*}, \\
H_{s}(x, t)+H_{i}(x, t) & =H^{*} .
\end{aligned}
$$


Equations (4) allow one to reduce equations (3) to infected populations only, so we finally obtain:

$$
\begin{aligned}
V_{j i}(x, t+1)= & \sigma_{j}(1-\tau) V_{j}^{*} \int_{-\infty}^{+\infty} \Lambda_{j} K_{j}(|x-y|) d y \\
& +\sigma_{a} V_{a}^{*} \int_{-\infty}^{+\infty} f_{v} \Lambda_{j} K_{a}(|x-y|) d y \\
& +\sigma_{j}(1-\tau) \int_{-\infty}^{+\infty}\left(1-\Lambda_{j}\right) K_{j}(|x-y|) V_{j i}(y, t) d y \\
V_{a i}(x, t+1)= & \sigma_{j} \tau V_{j}^{*} \int_{-\infty}^{+\infty} \Lambda_{a} K_{j}(|x-y|) d y+\sigma_{a} V_{a}^{*} \int_{-\infty}^{+\infty} \Lambda_{a} K_{a}(|x-y|) d y \\
& +\sigma_{j} \tau \int_{-\infty}^{+\infty}\left(1-\Lambda_{a}\right) K_{j}(|x-y|) V_{j i}(y, t) d y \\
& +\sigma_{a} \int_{-\infty}^{+\infty}\left(1-\Lambda_{a}\right) K_{a}(|x-y|) V_{a i}(y, t) d y \\
H_{i}(x, t+1)= & \sigma_{h} H^{*} \int_{-\infty}^{+\infty}\left(1+f_{h}\right) \Lambda_{h} K_{h}(|x-y|) d y \\
& +\sigma_{h} \int_{-\infty}^{+\infty}\left(1+\nu f_{h}\right)\left(1-\Lambda_{h}\right) K_{h}(|x-y|) H_{i}(y, t) d y
\end{aligned}
$$

Equations (5) are the major results of this section: they describe the three processes that occur between two successive generations. Equations (5) will be analyzed numerically and analytically. Model parameters are summarized in Table 1.

\section{Numerical results}

This section presents some numerical results using sample parameter values to illustrate the roles played by certain quantities in the advance of the infection. Results are shown for positive $x$ values beginning at 0 , with the wave moving in the positive direction from one time step to the next. In each resulting "generation" the infection spreads through the dispersal of infected hosts and vectors, which then begin the infection process in their new locations. In this way the maximal accumulated dispersal increases with each generation. Graphs in this section show the sizes of infected populations only; comparing results from different scenarios shows that model parameters affect not only the amplitude and speed of the traveling wave solutions, but also which population (juvenile vectors, adult vectors, or hosts) carries the leading edge of the infection. 
Table 1: Model parameters with values used to generate Figures 3-7. Where multiple values are given, Figures 3 and 4 use the value(s) before the comma, while Figures 5-7 use the value after the comma [t.s.=time step].

\begin{tabular}{c|lc}
\hline \hline Parm. & Definition & Values used \\
\hline$T$ & Time step & $2 \mathrm{wks}, 4 \mathrm{wks}$ \\
& Demographic parameters & \\
$\sigma_{j}$ & Survival prob./t.s. for juvenile vectors & $0.94,0.89$ \\
$\sigma_{a}$ & Survival prob./t.s. for adult vectors & $0.88,0.76$ \\
$\sigma_{h}$ & Survival prob./t.s. for hosts & $0.90,0.80$ \\
$f_{v}^{\text {max }}$ & Maximum [adult] vector fecundity/t.s. & 100 \\
$f_{h}^{\max }$ & Maximum host fecundity/t.s. & 1 \\
$\tau$ & Maturation prob./t.s. for [juvenile] vectors & $0.09 / 0.90,0.17$ \\
& Infection parameters & \\
$\beta_{1}$ & Infection rate/t.s. btw. hosts \& juvenile vectors & 0.001 \\
$\beta_{2}$ & Infection rate/t.s. btw. hosts \& adult vectors & 0.001 \\
$\nu$ & Prob. of vertical transmission in hosts & 0.5 \\
& Dispersal parameters & \\
$j$ & Mean dispersal distance/t.s. for juvenile vectors & 0.5 \\
$a$ & Mean dispersal distance/t.s. for adult vectors & 1.0 \\
$h$ & Mean dispersal distance/t.s. for hosts & 1.0 \\
\hline \hline
\end{tabular}

Figure 3 presents a representative set of results for a sample parameter set including a time step of 2 weeks. Here, as seen in the superposed graphs on the right showing the waves in the three populations after 20,26, and 30 generations, the host population carries the wavefront, followed closely by the juvenile vectors despite their lower dispersal ability, and finally the adult vectors.

We can investigate the role played by the maturation rate of the vectors by increasing the proportion of juveniles which mature at each time step. Figure 4 presents results based on a transition proportion of 0.9 , an order of magnitude higher than the baseline provided by Figure 3, where only 0.09 (9\%) mature each time step. The most immediately evident result is a marked change in the shape of the traveling wave for the juvenile vectors, as seen in the first graph in Figure 4; after an initial rise, the infection levels among juveniles level off, since the vast majority of infected juveniles in each generation become infected adults in the following generation. As illustrated in the comparative graphs on the right side of the table, the resulting depression in the levels of juvenile vector infection compared to those for adult vectors and hosts makes the waves in these latter two populations essentially identical. 
We can also investigate the quantitative effects of changing the time step or generation time; however, since those model parameters representing probabilities or proportions of events (birth, death, infection, etc.) occurring within a single generation, a change in the time step necessitates changes in many of the other parameters. For a proportion $p$ derived from an average rate $r$ (or, inversely, average waiting time $1 / r$ ), the conversion to a proportion or probability uses an exponential law:

$$
p=1-\exp (-r T)
$$

where $T$ is the time step. So, for example, if juvenile vectors mature at a rate of $r=0.047 / w k$, then when $T=2 w k$, the maturation proportion

$$
\tau=1-\exp (-0.047 * 2)=0.09 \text {. }
$$

When $T=4 w k$, however, we have

$$
\tau=1-\exp (-0.047 * 4)=0.17 \text {. }
$$

The change in generation time therefore changes several parameters (see Figure 5), but in this case produces results consistent with those in Figure 3. This is reassuring since the change in time step is largely a modeling decision in this case, and the consistency in results shows that the decision does not have a major impact on predictions. (The system's qualitative behavior is a function of parameter values, including the time step, but the discrete-time model is an approximation of a system with discrete events which occur in continuous time, so one hopes that this discretization does not affect the model's ability to describe the biological system's qualitative behavior).

Finally, Figures 6 and 7 investigate the role played by vertical transmission in the spread of infection. The graphs in these figures show results for a full spectrum of values for the vertical transmission proportion $v$, from $0 \%$ to $100 \%$. Figure 6 shows that, predictably, the infection spreads faster as the vertical transmission proportion increases. The comparative graphs in Figure 7, however, show a less obvious result: when vertical transmission is unlikely (approximately $v<0.3$ for our parameter values), the wavefront is carried by the vectors (with juveniles and vectors in close alignment, and much higher incidence than in the host population), but when vertical transmission is more likely it is instead the hosts that carry the wavefront, as the vertical transmission amplifies the spread of infection in the host population so much that it becomes a much stronger source of infection (for vectors) than the vectors are (for hosts). 


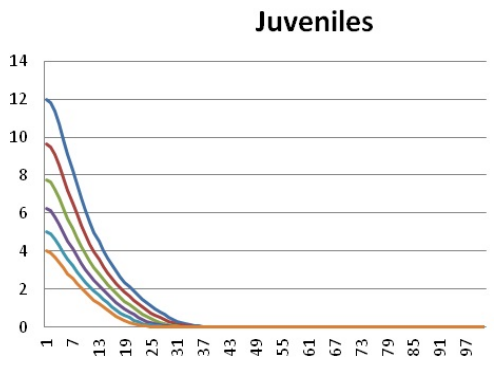

(a) Infected juvenile vector density after (from bottom to top) 20, 22, 24, $26,28,30$ time steps.

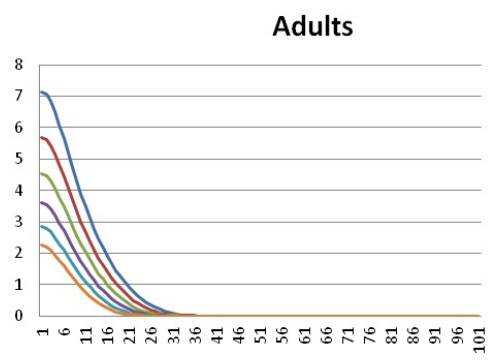

(c) Density of infected adult vectors after (from bottom to top) 20, 22, 24, 26, 28, 30 time steps.

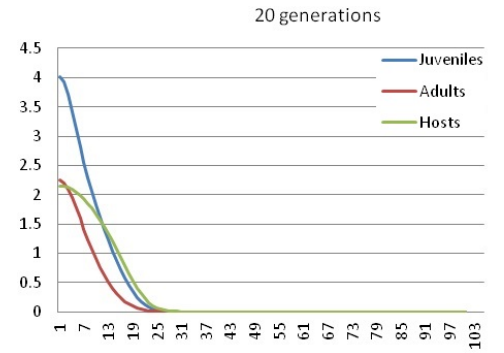

(b) Densities of infected populations after 20 time steps.

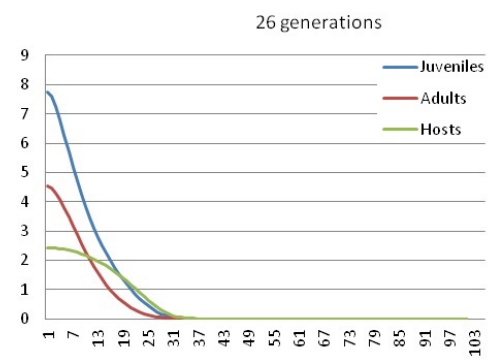

(d) Densities of infected populations after 26 time steps.

30 generations

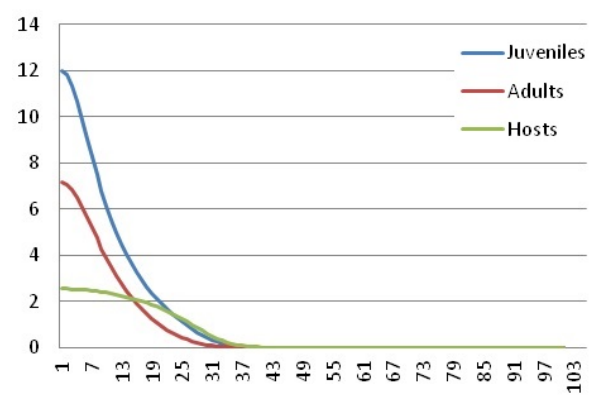

(f) Densities of infected populations after 30 time steps.

(e) Density of infected hosts after (from bottom to top) 20, 22, 24, 26, 28, 30 time steps.

Hosts

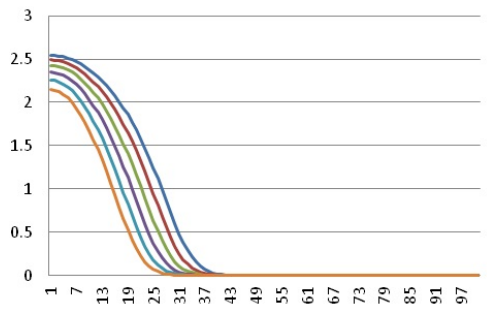

Figure 3: Travelling waves of infected juvenile vectors, adult vectors and hosts, respectively, vs. dispersal distance (in units of mean adult dispersal distance per time step) for slow vector maturation, $\tau=0.09$. Other values are as in Table 1 . 
Juveniles

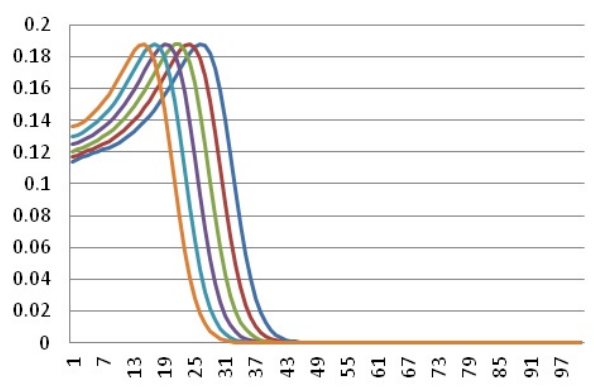

(a) Infected juvenile vector density after (from bottom to top) $20,22,24,26,28,30$ time steps.

\section{Adults}

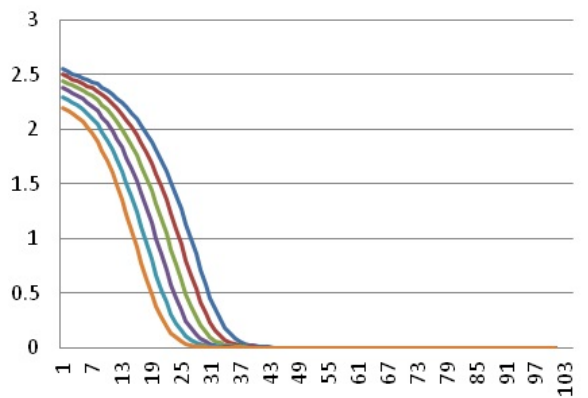

(c) Density of infected adult vectors after (from bottom to top) $20,22,24,26,28,30$ time steps.

Hosts

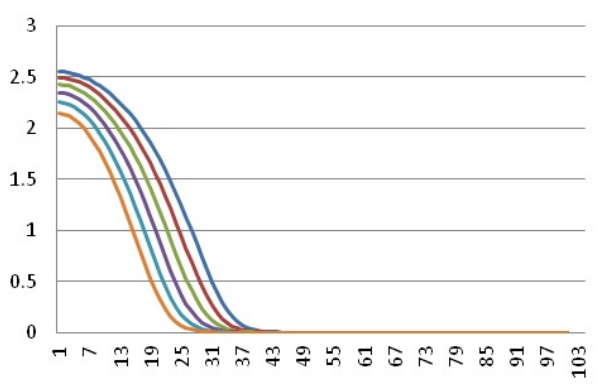

(e) Density of infected hosts after (from bottom to top) $20,22,24,26,28,30$ time steps.

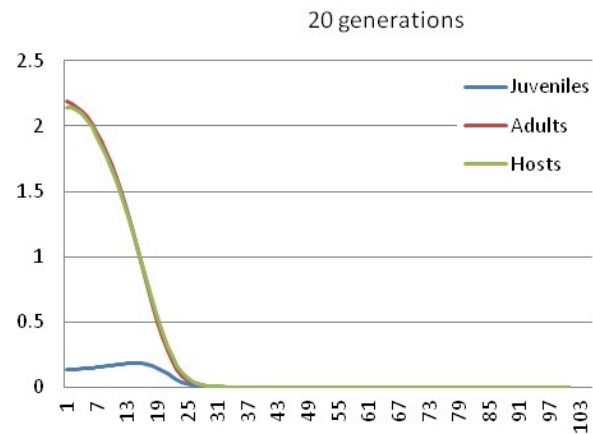

(b) Densities of infected populations after 20 time steps.

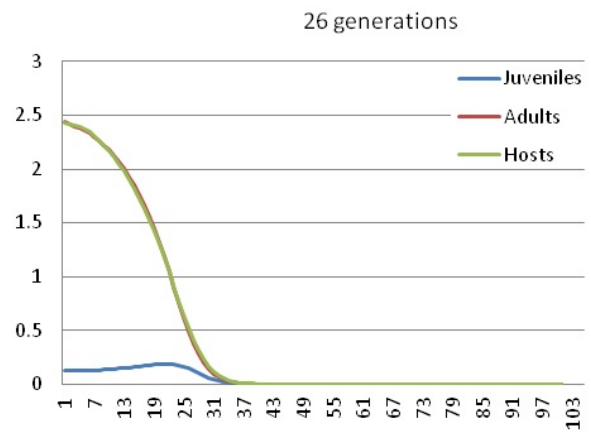

(d) Densities of infected populations after 26 time steps.

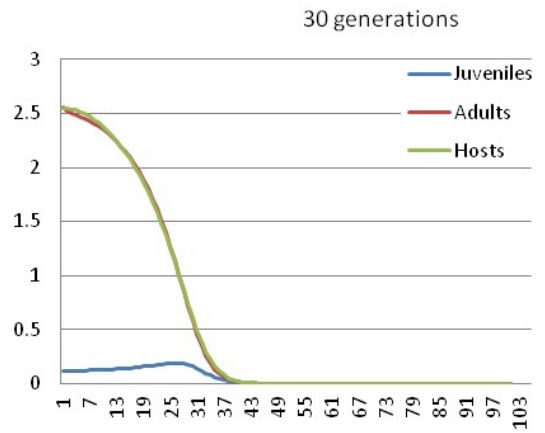

(f) Densities of infected populations after 30 time steps.

Figure 4: Travelling waves of infected juvenile vectors, adult vectors and hosts, respectively, vs. dispersal distance (in units of mean adult dispersal distance per time step) for fast vector maturation, $\tau=0.90$. Other values are as in Table 1 . 


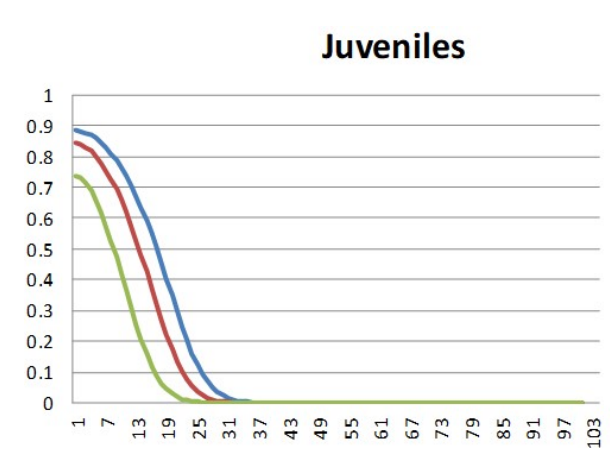

(a) Density of infected juvenile vectors after (from bottom to top) 20, 26, 30 time steps.

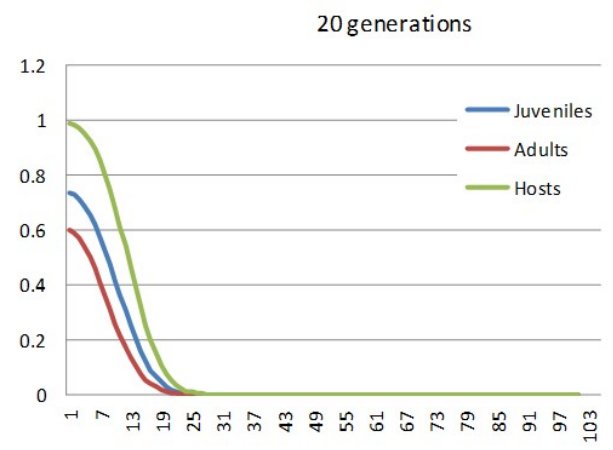

(b) Densities of infected populations after 20 time steps.

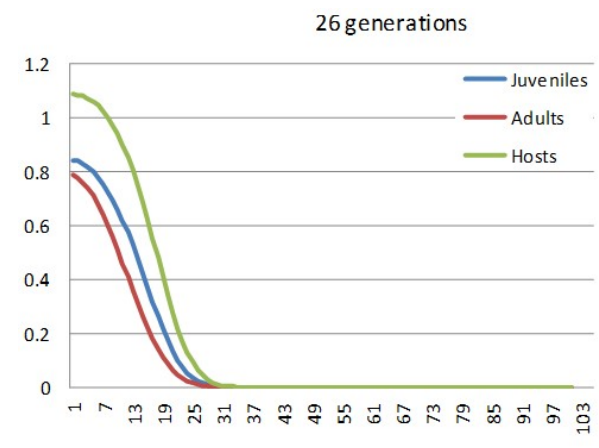

(d) Densities of infected populations after 26 time steps.

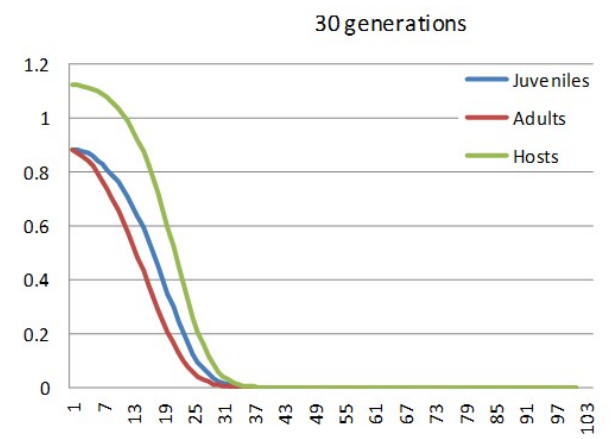

(f) Densities of infected populations after 30 time steps.

Figure 5: Travelling waves of infected juvenile vectors, adult vectors and hosts, respectively, vs. dispersal distance (in units of mean adult dispersal distance per time step) for a longer (4 weeks) time step. Other values are as in Table 1. 

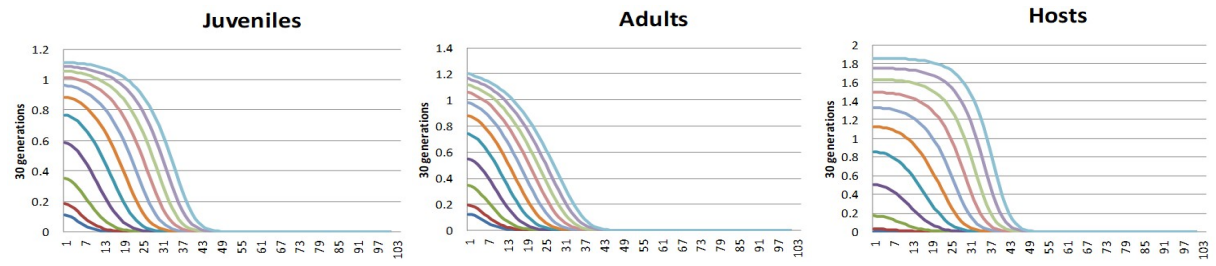

(a) Density of infected juvenile (b) vectors as $\nu$ runs (from top to bottom) from 0 to 1 in increments of 0.1 .

(b) Density of infected adult (c) vectors as $\nu$ runs (from top to bottom) from 0 to 1 in increments of 0.1 . c) Density of infected hosts as $\nu$ runs (from top to bottom) from 0 to 1 in increments of 0.1 .

Figure 6: Travelling waves of infected juvenile vectors, adult vectors and hosts, respectively, vs. dispersal distance (in units of mean adult dispersal distance per time step) as vertical T. cruzi transmission $\nu$ varies, after 30 time steps. Parameters are as in Table 1.

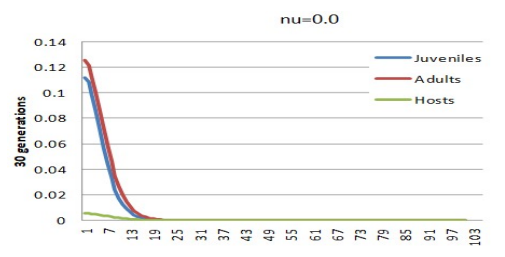

(a) Densities of infected populations without vertical transmission.

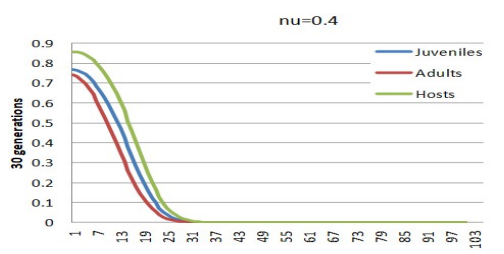

(c) Densities of infected populations with $40 \%$ vertical transmission.

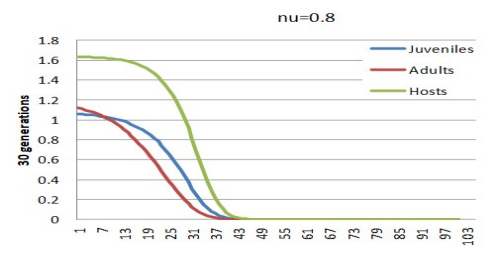

(e) Densities of infected populations with $80 \%$ vertical transmission.

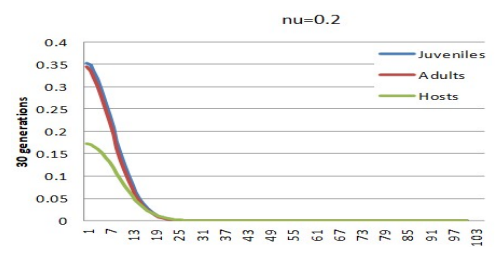

(b) Densities of infected populations with $20 \%$ vertical transmission.

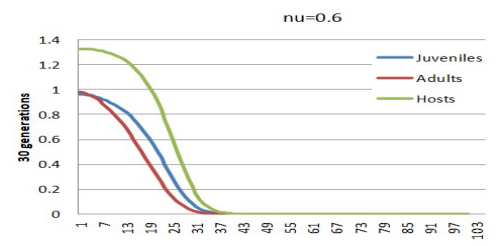

(d) Densities of infected populations with $60 \%$ vertical transmission.

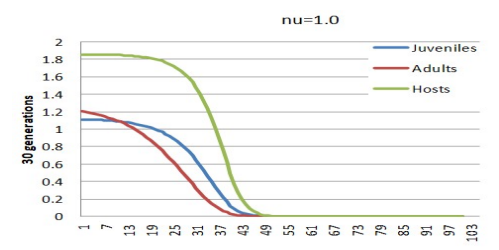

(f) Densities of infected populations with $100 \%$ vertical transmission.

Figure 7: Superimposed travelling waves of infected juvenile vectors, adult vectors and hosts, respectively, vs. dispersal distance (in units of mean adult dispersal distance per time step) after 30 time steps, for various vertical transmission proportions. Parameters are as in Table 1. 


\section{Analytical results}

Equations (5) can be rewritten using Neubert-Caswell notation [6]: writing

$$
\begin{aligned}
A_{N_{i}}= & {\left[\begin{array}{ccc}
\sigma_{j}(1-\tau) \Lambda_{j} & \sigma_{a} f_{v} \Lambda_{j} & 0 \\
\sigma_{j} \tau \Lambda_{a} & \sigma_{a} \Lambda_{a} & 0 \\
0 & 0 & \sigma_{h}\left(1+f_{h}\right) \Lambda_{h}
\end{array}\right], } \\
B_{N_{i}}= & {\left[\begin{array}{ccc}
\sigma_{j}(1-\tau)\left(1-\Lambda_{j}\right) & 0 & 0 \\
\sigma_{j} \tau\left(1-\Lambda_{a}\right) & \sigma_{a}\left(1-\Lambda_{a}\right) & 0 \\
0 & 0 & \sigma_{h}\left(1+\nu f_{h}\right)\left(1-\Lambda_{h}\right)
\end{array}\right], }
\end{aligned}
$$

and the dispersal kernel matrix:

$$
K(x)=\left[\begin{array}{lll}
K_{j}(x) & K_{a}(x) & K_{h}(x) \\
K_{j}(x) & K_{a}(x) & K_{h}(x) \\
K_{j}(x) & K_{a}(x) & K_{h}(x)
\end{array}\right]
$$

we obtain the equation:

$$
N_{i}(x, t+1)=\int_{-\infty}^{+\infty}\left[K(|x-y|) \circ A_{N_{i}}\right] N^{*} d y+\int_{-\infty}^{+\infty}\left[K(|x-y|) \circ B_{N_{i}}\right] N_{i}(y, t) d y
$$

where the state vector is $N_{i}(x, t)=\left(V_{j i}, V_{a i}, H_{i}\right)^{T}(x, t)$ and $N^{*}=\left(V_{j}^{*}, V_{a}^{*}, H^{*}\right)^{T}$ and "o" is the term-by-term product.

We define:

$$
\begin{aligned}
& \phi\left(V_{j i}, V_{a i}, H_{i}\right)=\sigma_{j}(1-\tau) \Lambda_{j} V_{j}^{*}+\sigma_{a} f_{v} \Lambda_{j} V_{a}^{*}, \\
& \psi\left(V_{j i}, V_{a i}, H_{i}\right)=\sigma_{j} \tau \Lambda_{a} V_{j}^{*}+\sigma_{a} \Lambda_{a} V_{a}^{*} \\
& \chi\left(V_{j i}, V_{a i}, H_{i}\right)=\sigma_{h}\left(1+f_{h}\right) \Lambda_{h} H^{*} .
\end{aligned}
$$

It is clear that these three functions are of class $C^{1}\left(\mathbb{R}^{3}\right)$. Taylor expansion of the vectorial function $\Phi=(\phi, \psi, \chi)^{T}$ and in the neighborhood of $(0,0,0)$ gives:

$$
A_{N_{i}} N^{*}=\left.\Phi\right|_{(0,0,0)}+\left.J_{\Phi}\right|_{(0,0,0)}\left(V_{j i}, V_{a i}, H_{i}\right)^{T}+\left.\left(R_{1}, R_{2}, R_{3}\right)^{T}\right|_{\left(V_{j i}, V_{a i}, H_{i}\right)}
$$

where $\lim _{\left(V_{j i}, V_{a i}, H_{i}\right) \rightarrow(0,0,0)} R_{k}\left(V_{j i}, V_{a i}, H_{i}\right)=0$ for $k=1,2,3$ and $J_{\Phi}$ is the Jacobian of $\Phi$ defined by:

$$
J_{\Phi}=\left[\begin{array}{ccc}
-\sigma_{a} f_{v} V_{a}^{*} \Lambda_{j} & -\sigma_{a} f_{v} V_{a}^{*} \Lambda_{j} & \beta_{1}\left[\sigma_{j}(1-\tau) V_{j}^{*}+\sigma_{a} f_{v} V_{a}^{*}\right]\left(1-\Lambda_{j}\right) \\
0 & 0 & \beta_{2}\left[\sigma_{j} \tau V_{j}^{*}+\sigma_{a} V_{a}^{*}\right]\left(1-\Lambda_{a}\right) \\
\beta_{1} g & \beta_{2} g & -\sigma_{h} f_{h} H^{*} \Lambda_{h}
\end{array}\right],
$$


where $g=\sigma_{h}\left(1+f_{h}\right) H^{*}\left(1-\Lambda_{h}\right)$. But:

$$
\left.\Phi\right|_{(0,0,0)}=0
$$

and:

$$
\left.J_{\Phi}\right|_{(0,0,0)}=\left[\begin{array}{ccc}
0 & 0 & \beta_{1}\left[\sigma_{j}(1-\tau) V_{j}^{*}+\sigma_{a} f_{v}^{\max } V_{a}^{*}\right] \\
0 & 0 & \beta_{2}\left[\sigma_{j} \tau V_{j}^{*}+\sigma_{a} V_{a}^{*}\right] \\
\beta_{1} \sigma_{h}\left(1+f_{h}^{\max }\right) H^{*} & \beta_{2} \sigma_{h}\left(1+f_{h}^{\max }\right) H^{*} & 0
\end{array}\right] .
$$

By the linear conjecture, the invasion speed of the system described by the integrodifference equation (7) is the same as that of the system:

$$
N_{i}(x, t+1)=\int_{-\infty}^{+\infty} K(|x-y|) \circ\left[\left.J_{\Phi}\right|_{(0,0,0)}+B_{(0,0,0)}\right] N_{i}(y, t) d y .
$$

Following the Neubert-Caswell theorem [6], the matrix of the moment generating functions can be written via (5) and (2):

$$
M(s)=\left[\begin{array}{ccc}
\frac{1}{1-j^{2} s^{2}} & \frac{1}{1-a^{2} s^{2}} & \frac{1}{1-h^{2} s^{2}} \\
\frac{1}{1-j^{2} s^{2}} & \frac{1}{1-a^{2} s^{2}} & \frac{1}{1-h^{2} s^{2}} \\
\frac{1}{1-j^{2} s^{2}} & \frac{1}{1-a^{2} s^{2}} & \frac{1}{1-h^{2} s^{2}}
\end{array}\right] .
$$

The asymptotic invasion speed depends on the largest eigenvalue of the matrix $H(s)=\left[\left.J_{\Phi}\right|_{(0,0,0)}+B_{(0,0,0)}\right] \circ M(s)$, which is defined by the relation:

$$
H(s)=\left[\begin{array}{ccc}
\frac{\sigma_{j}(1-\tau)}{1-j^{2} s^{2}} & 0 & \frac{\beta_{1}\left[\sigma_{j}(1-\tau) V_{j}^{*}+\sigma_{a} f_{\max }^{v} V_{a}^{*}\right]}{1-h^{2} s^{2}} \\
\frac{\sigma_{j} \tau}{1-j^{2} s^{2}} & \frac{\sigma_{a}}{1-a^{2} s^{2}} & \frac{\beta_{2}\left[\sigma_{j} \tau V_{j}^{*}+\sigma_{a} V_{a}^{*}\right]}{1-h^{2} s^{2}} \\
\frac{\beta_{1} \sigma_{h}\left(1+f_{h}^{\max }\right) H^{*}}{1-j^{2} s^{2}} & \frac{\beta_{2} \sigma_{h}\left(1+f_{h}^{\max }\right) H^{*}}{1-a^{2} s^{2}} & \frac{\sigma_{h}\left(1+\nu f_{h}^{\max }\right)}{1-h^{2} s^{2}}
\end{array}\right] .
$$

Indeed, if $\rho(s)$ is the largest eigenvalue of the matrix $H(s)$, then the asymptotic invasion speed of the biological system described by equations (7) satisfies:

$$
c^{*}=\min _{0<s<\hat{s}}\left(\frac{1}{s} \ln \rho(s)\right),
$$

where $\hat{s}=\min \left(\frac{1}{j}, \frac{1}{\alpha}, \frac{1}{h}\right)$ in order to avoid singularities at $s=j, s=\alpha, s=h$. This expression can be evaluated numerically for specific parameter values.

A question arises from this calculation, however: to which wave does this speed correspond, i.e., in which of the three populations does the infection spread 
with this speed? Or does it represent some combination of the three, as might be suggested by an eigenvector of $H(s)$ ? Asked another way, what contribution does each species make to the invasion speed? The Neubert-Caswell measure was originally developed for single populations [6], and the extension developed here for multiple populations leaves this question open and deserving further study.

\section{Conclusion}

This work develops and illustrates a spatially explicit model for the sylvatic transmission of the parasite Trypanosoma cruzi. The model can easily be adapted to represent other vector-borne infections. The model, consisting of three coupled integrodifference equations, describes the spread (in a single spatial dimension) of $T$. cruzi infection among dispersing populations of juvenile and adult vectors, and hosts, and to develop an expression for the asymptotic invasion speed.

The numerical results presented here using sample parameter values show the influences of some of the demographic processes on the invasion speed, most notably how vertical (transplacental) transmission in hosts changes which species carries the wavefront. Namely, for high enough vertical transmission (about 30\% for our parameter estimates) hosts carry the wavefront, rather than vectors. It remains to develop more precise estimates of the processes under study in order to derive an estimate of invasion speed which can be compared with results of studies using other modeling approaches (e.g., [1]). These results will also permit the calculation and study of spatial as well as temporal variation in the infection's basic reproductive number, $R_{0}$.

\section{Acknowledgements}

This research was supported by a grant from the Fulbright Program, grant 68435333.

\section{References}

[1] B.A. Crawford, C.M. Kribs-Zaleta, G. Ambartsoumian, Invasion speed in cellular automaton models for $\mathrm{T}$. cruzi vector migration, Bulletin of Mathematical Biology 75(2013), no. 7, 1051-1081. doi: $10.1007 /$ s11538-013-9840-7

Rev.Mate.Teor.Aplic. (ISSN print: 1409-2433; online: 2215-3373) Vol. 27(1): 73-92, Jan-Jun 2020 
[2] C.M. Kribs-Zaleta, Estimating contact process saturation in sylvatic transmission of Trypanosoma cruzi in the United States, PLoS Negl Trop Dis 4(2010), no. 4, e656, 1-14. doi: 10.1371 / journal . pntd. 0000656

[3] F.X. Lescure, G. Le Loup, H. Freilij, M. Develoux, L. Paris, G. Pialoux, Chagas disease: changes in knowledge and management, Lancet Infect Dis 10(2010), no. 8, 556-570. doi: 10 .1016/S1473-3099 (10) 70098-0

[4] Médecins Sans Frontières. The fight against Chagas disease: time to focus on patients. 09 July 2009. https://www.msf.org/ fight-against-chagas-time-focus-patients, accessed Mar/03/2012.

[5] F. Menu, M. Ginoux, E. Rajon, C.R. Lazzari, J.E. Rabinovich, Adaptive developmental delay in Chagas disease vectors: an evolutionary ecology approach, PLoS Negl Trop Dis 4(2010), no. 5, e691, 1-10. doi: $10.1371 /$ journal.pntd.0000691

[6] M.G. Neubert, H. Caswell, Demography and dispersal: calculation and sensitivity analysis of invasion speed for structured populations, Ecology 81(2000), no. 6, 1613-1628. doi: 10 .2307/177311

[7] World Health Organisation. Chagas disease (American trypanosomiasis). Fact sheet 340, June 2010. https: / / www. who. int/chagas / disease/en/, accessed Mar/03/2012. 